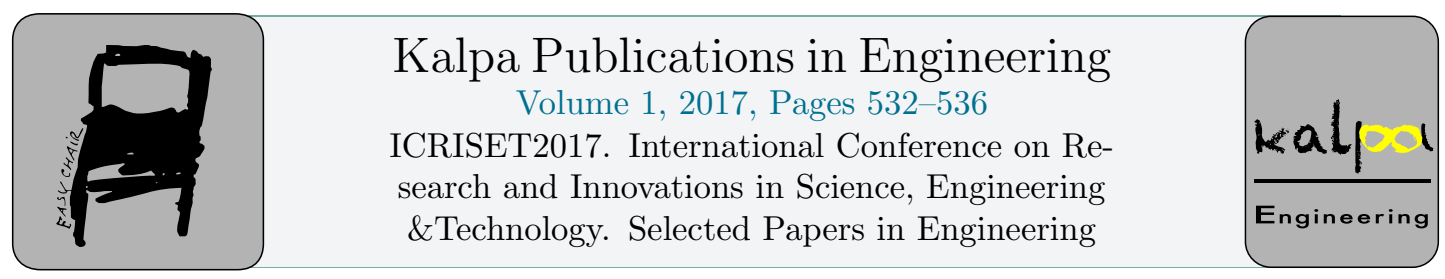

\title{
Beacon Based Positioning and Tracking with SOS
}

\author{
Hiren Patel1, Robinson Paul1, Dr. Vipul Shah2 \\ ${ }^{1}$ Electronics \& Communication Department \\ BVM Engineering College \\ Anand, India \\ ${ }^{2}$ Instrumentation \& Control Engineering Department \\ Faculty of Technology \\ Nadiad, India
}

\begin{abstract}
The use of wireless sensor network is increasing due to its effectiveness over wired systems so far as large area coverage is of concern. Wireless sensor network is used to collect data and distribute controls over network but here we are proposing solution to positioning system using wireless network. It becomes essential in many situations to locate the position of the nodes or persons in field. We are using a MCU based system to facilitate the user to identify the position of the deployed nodes in the field.
\end{abstract}

\section{Introduction}

Wireless Sensor networks is gaining the importance in field of test and measurement over the network due its coverage benefit as well as cost effective. Wireless sensor networks are resource limited and more focused on energy conserving based design. There are various ways to conserve energy in wireless sensor network by managing firmware to work with limited resoures[1]a. Wireless sensor networks Networked sensors can function in wide variety of ways to make it useful in various applications.

We have devised a system to identify the position of the node in the network for locating during SOS (signal of distress). The situation may arise in many situations where person or object is to be traced when there is alarming situation.

We have considered a situation when the troops are deployed in forest for search and rescue operations as shown in fig. 1. In such scenario when an object or activity in a suspicious region is detected it is required to call the force to act upon at the detected region. The hand held devices such 
as mobiles may not be useful as such regions be deprived of the networks or internet access. So the gadget which can work without mobile network and internet access mush be devised.

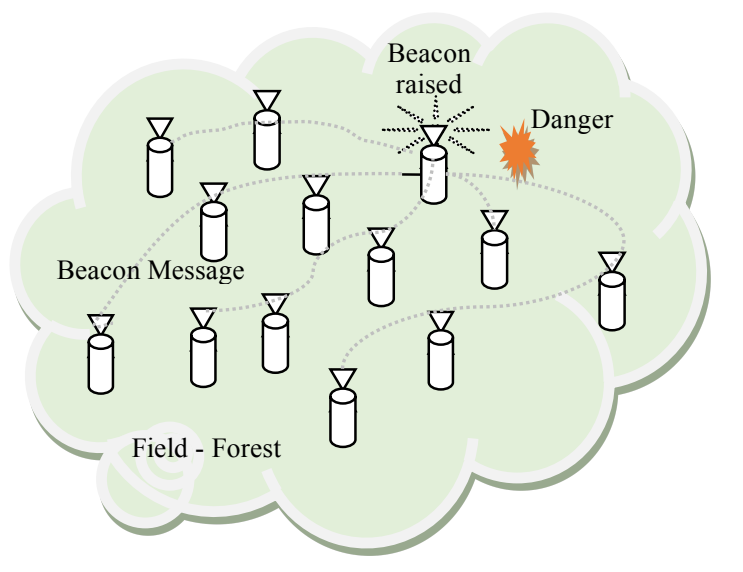

Fig. 1. Scenario of troops deployed in forest for search and rescue operation

Before the troops may arrive at the site it is very essential to locate the site. So beacon is used to inform troops to publish the location of site with coordinates. The most essential component of the system is positioning device. There are various ways to locate a node in the network one of which is geographic routing[2]. It may use Global Positioning System (GPS)[3] or network to estimated location by signal strength[4]. The GPS based locations are more accurate than network estimated locations but it adds cost to the system[5].

Once the position is identified it is required to send the coordinates other nodes. There are many ways to route the data to the nodes in the network[6] and form the clusters[7]. We are not focusing on the routing and clustering to form the network here in this paper but though it is very essential part of the system.

The coordinate received are used to find the direction and the distance to reach the site. This is done at the received node and the distance is plotted on the screen to navigate through. To form the plot for navigation it is required to have the raw region plot with details. The path to the destination can be plotted considering the details and obstacles. Here we have considered the forest area or open land where the obstacle are ignored at initial stage of development. The offline land maps may be used to plot the exact details of land.

\section{System Overview}

The system composed of hardware including MCU with wireless connectivity and screen to plot the navigation map. The nodes will be interconnected with wireless media.

The MCU used is Atmega32 which is advance RISC 8-bit microcontroller[8]ate as shown in fig. 2. The role of MCU is to send the beacon with coordinates to inform the rest of the nodes about its location. It also identify the location from coordinates and plot it on to the screen, graphical LCD $128 \times 64$, for navigation.

The keypad is for the SOS switch and other setting to perform in the system. NRF24L01 is used for the wireless connectivity. It is low power RF transceiver module with frequency of $2.4 \mathrm{GHz}$ and programmable power range[9][10]. 


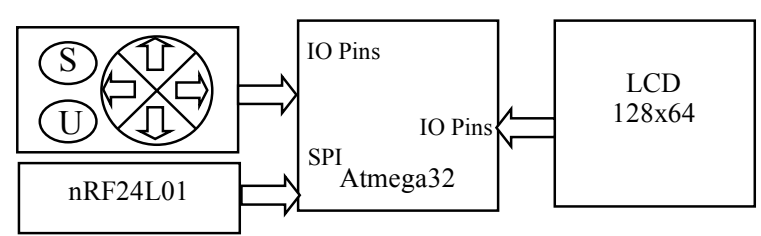

Fig. 2. Hardware Setup

\section{Algorithm For Positioning}

Fig. 3. Shows the algorithm for the application. There are two tasks to be performed by the program: listen to the beacon continuously and to poll the switch for generating the SOS beacon. Listening to the beacon is a continuous task which a node has to perform in order to hear beacon call.

The other task is to check for the SOS switch(S). If the troop member detects threat he will press the switch to call for the backup team. The switch input will generate the interrupt to the main routing of listening to the beacon and switch to the transmission mode to send the beacon signal to the trooper in the region.

\section{A. Locating the position of node}

If the network is distributed over a wide area in field the hop-to-hop routing is carried out by the routing protocol so that the beacon reaches to the end node in the field. Every node passes the connectivity-packet in network after every small interval of time. So if any node stops receiving the packet it means it is not in the network so it will be informed to be in the radio range to stay connected with others.

Each node maintains the connectivity table rather neighbor routing table. The inter distance between nodes is estimated by the radio range. First of all the node transmits the signal to neighboring nodes to respond with their IDs with lowest radio range. The received responses will form the table of nearest neighbors to that node. Likewise node will perform similar action with increased level of radio power to form total 3 such tables.

Finally all such tables are used to map the nodes in the field to find physical location of node relative to each other. If the transmission power range is $1000 \mathrm{~m}$ at full power than dividing the range in 3 parts depending upon power will give approximate distance of coverage. Thus the node displacement can be approximately estimated using this technique.

\section{B. Tracing the node}

Once the network of approximately displaced nodes with IDs are known the mapping can be done. When a node sends beacon for call it also sends it's ID. From the ID, location of node can be estimated from mapped node table. Mathematically the distance is calculated from itself to the site of beacon.

The map formed after the calculation of distance and position estimation will give direction and distance toward the target. As the node starts moving toward the destination its relative distance decreases. The map is not update on the go with the movement of node as it consumes lot of power for the gadget in communicating the position table. A switch (U) is provided on the gadget to request the update of map if node is lost while migrating.

\section{Map-Update mode}

The update request will initiate update-map routine to replot the nodes in field. The process here is similar to that performed after listening beacon. The update-map is passed on to the nodes in the 
network, if any other request is raised prior than it is dropped to avoid redundancy in network. So inspite of multiple simultaneous requests only one request is propagated in the network, duplicate requests are dropped.

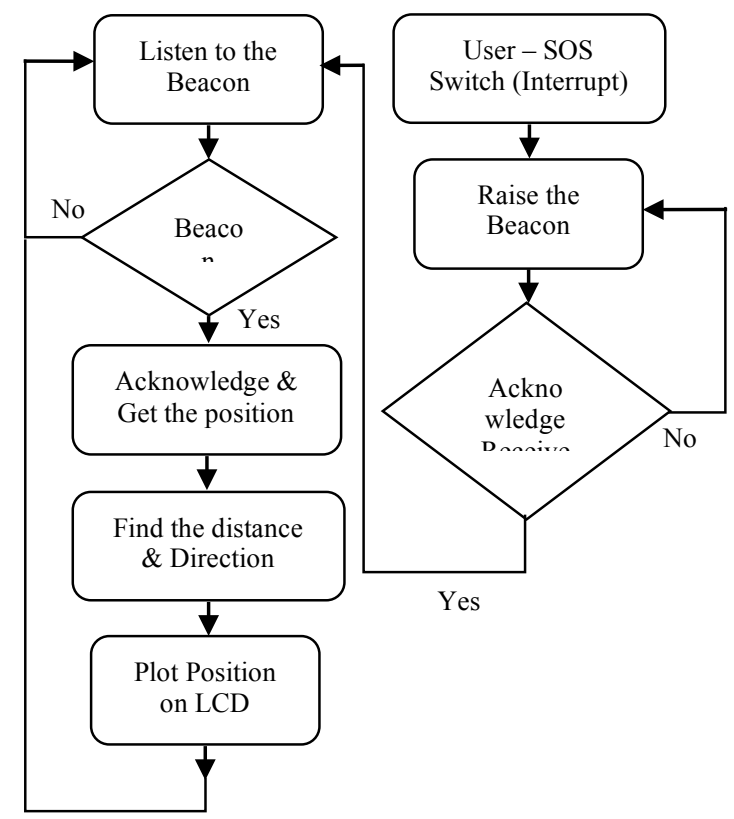

Fig. 3. Algorithm for the application

On receiving the request for the update of map, all nodes gather the information of the nearest to farthest neighbors as done previously and passed on to the sink in network. The sink will calculate the distance and does the mapping of nodes in the network on to the field.

\section{Outcome and Conclusion}

The mapping of the nodes on the field becomes tedious if the network density is very high and huge. As the first stage of mapping is collecting the connectivity data from all the nodes in the network it requires all the nodes to check for nodes in its radio range. Secondly it has to form table of connected nodes to it with displacement data to classify as nearest to farthest nodes. All this process consumes time and power of node. So frequent update of mapping is not practical in network to conserve node energy.

Making use of GPS in the system makes it more precise measurement and plotting nodes on the field. But the system with GPS adds cost to the system simultaneously makes it network independent too.

This system can be very useful and cost-effective for the purpose of getting the approximate information about the target. If the field is open land, then just by knowing approximate distance and direction can meet the job. 


\section{Future Work}

The proposed system functions to generate the SOS call and get the map to reach the destination. More add-on functionality can be made to increase features such as detecting the health of the user, movement detection or other environmental conditions. Integrating all this features will enable the sink to detect the exact condition at the node.

\section{REFERENCES}

[1] H. Patel, "A Review on Energy consumption and conservation techniques for Sensor node in WSN," 2016.

[2] H. Zhang and H. Shen, "Energy-Efficient Beaconless Geographic Routing in Wireless Sensor Networks," IEEE Trans. Parallel Distrib. Syst., vol. 21, no. 6, pp. 881-896, 2010.

[3] A. E. Zonouz et al., "Reliability-Oriented Single-Path Routing Protocols in Wireless Sensor Networks," IEEE Sens. J., vol. 14, no. 11, pp. 4059-4068, 2014.

[4] T. Adnan, S. Datta, and S. MacLean, "Effcient and Accurate Range-based Sensor Network Localization," Procedia Comput. Sci., vol. 10, pp. 405-413, 2012.

[5] G. Anastasi, M. Conti, M. Di Francesco, and A. Passarella, "Energy conservation in wireless sensor networks: A survey," Ad Hoc Networks, vol. 7, no. 3, pp. 537-568, 2009.

[6] C. Schurgers and M. B. Srivastava, "Energy Efficient Routing in Wireless Sensor Network," Mil. Commun. Conf. MILCOM 2001. Commun. Network-Centric Oper. Creat. Inf. Force., vol. 1, pp. 357-361, 2001.

[7] Z. Yong and Q. Pei, "A Energy-Efficient Clustering Routing Algorithm Based on Distance and Residual Energy for Wireless Sensor Networks," Procedia Eng., vol. 29, pp. 1882-1888, 2012.

[8] Atmel Corporation, Programmable ATmega32 ( L ). 2011.

[9] R. P. Narayanan, T. V. Sarath, and V. V. Vineeth, "Survey on Motes Used in Wireless Sensor Networks: Performance \&amp;amp; Parametric Analysis," Wirel. Sens. Netw., vol. 8, no. 4, pp. 67-76, 2016.

[10] P. Specification and K. Features, Product Specification v1.0, no. September. 2008. 